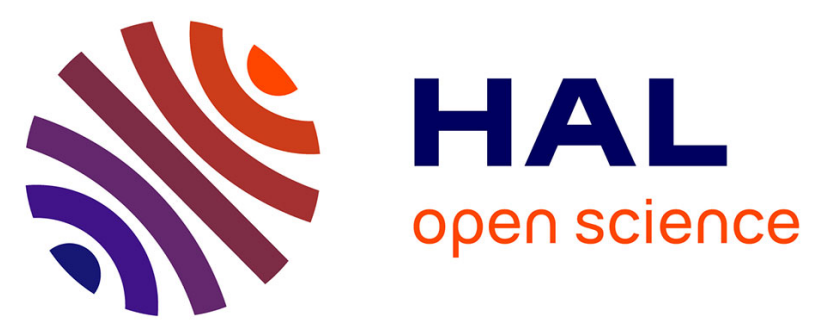

\title{
Space and time resolved measurement of surface magnetic field in high intensity short pulse laser matter interactions
}

Pierre Forestier-Colleoni, Dimitri Batani, Frédéric Burgy, Dario Del Sorbo, Fanny Froustey, Sébastien Hulin, Emmanuel d'Humières, Katarzyna

Jakubowska, Laurent Merzeau, Konstantin Mishchik, et al.

\section{To cite this version:}

Pierre Forestier-Colleoni, Dimitri Batani, Frédéric Burgy, Dario Del Sorbo, Fanny Froustey, et al.. Space and time resolved measurement of surface magnetic field in high intensity short pulse laser matter interactions. Physics of Plasmas, 2019, 26 (7), pp.072701. 10.1063/1.5086725 . hal-02271045

\section{HAL Id: hal-02271045 \\ https://hal.science/hal-02271045}

Submitted on 26 Aug 2019

HAL is a multi-disciplinary open access archive for the deposit and dissemination of scientific research documents, whether they are published or not. The documents may come from teaching and research institutions in France or abroad, or from public or private research centers.
L'archive ouverte pluridisciplinaire HAL, est destinée au dépôt et à la diffusion de documents scientifiques de niveau recherche, publiés ou non, émanant des établissements d'enseignement et de recherche français ou étrangers, des laboratoires publics ou privés. 


\title{
Space and time resolved measurement of surface magnetic field in high intensity short pulse laser matter interactions
}

\author{
Pierre Forestier-Colleoni, ${ }^{1}$ Dimitri Batani, ${ }^{1,}$ a) Frédéric Burgy, ${ }^{1}$ Dario Del Sorbo, ${ }^{1}$ Fanny Froustey, ${ }^{1}$ Sébastien \\ Hulin, ${ }^{1}$ Emmanuel d'Humières, ${ }^{1}$ Katarzyna Jakubowska, ${ }^{2}$ Laurent Merzeau, ${ }^{1}$ Konstantin Mishchik, ${ }^{1}$ Daniel \\ Papp, $^{3}$ and João Jorge Santos ${ }^{1, b)}$ \\ 1) Univ. Bordeaux, CNRS, CEA, CELIA (Centre Lasers Intenses et Applications), UMR 5107, F-33405 Talence, \\ France \\ ${ }^{2)}$ Institute of Plasma Physics and Laser Microfusion, 01-497 Warsaw, Poland \\ ${ }^{3)}$ ELI-ALPS, ELI-HU Non-Profit Ltd., Dugonics ter 13, Szeged 6720, Hungary
}

(Dated: 7 June 2019)

Magnetic fields produced by the interaction of intense laser beams on the surface of flat solid targets have been characterized. Laser probe polarimetry diagnosed the spatial and temporal evolution of magnetic field by measuring the changes in the probe beam polarization due to Cotton-Mouton and Faraday effects at different times in respect to a pump laser pulse. Results show that 1 ps after the interaction of the pump laser with the target, a magnetic field of the order of a few MG is already present over a region $\approx 150 \mu \mathrm{m}$ in diameter centered around the interaction spot. From the spatial and temporal evolutions of the magnetic field, we infer information on the resistivity of the material, showing evidence of a strongly magnetized resistivity.

\section{INTRODUCTION}

Strong magnetic fields are created during the interaction of laser beams with solid targets. Theoretical ${ }^{1}$, numerical ${ }^{2,3}$ and experimental studies ${ }^{4-6}$ were performed in the past 40 years to understand the creation mechanisms and the impact of these fields on plasma dynamics. More recently, many researches have focused on fast electron beam generation in ultra-highintensity laser interaction (for instance in the context of fast ignition) and on magnetic field effects in this regime ${ }^{7}$. It was shown that the magnetic field at the surface of the target can impact the fast electron beam propagation and it is probably at the origin of the large initial divergence of the electron beam ${ }^{3}$.

Several experiments were performed in order to characterize these magnetic fields. Borghesi et al. ${ }^{4}$ measured the magnetic field at the surface of the irradiated target by looking at the change of polarization of a probe beam at grazing incidence to the target. This change of polarization is induced by the magnetic field, created by the thermoelectric effect, parallel to the propagation of the probe beam. Unfortunately, the grazing incidence angle did not allow measuring the magnetic field closer than $40 \mu \mathrm{m}$ from the target surface.

A more recent study by Pisarczyk et al. ${ }^{8}$ used interferopolarimetric measurements (Faraday rotation) to assess the impact of fast electrons on the generation of a spontaneous magnetic field in a laser-driven plasma. They observed a magnetic field up to $20 \mathrm{MG}$ inside the pre-plasma and inferred the fast electron beam current responsible for this magnetic field.

Tatarakis et al. ${ }^{5}$ and Gopal et al. ${ }^{9}$ measured the change of polarization produced by the Faraday effect on the selfgenerated harmonics of the laser. This allowed to obtain information about the magnetic field deeper inside the plasma,

\footnotetext{
a) Also at National Research Nuclear University MEPhI, Department of Plasma Physics - Moscow, Russia.

b)Electronic mail: joao.santos@u-bordeaux.fr
}

but without information about the path of the harmonics inside the plasma (i.e. the exact position of harmonics generation).

Laser produced protons were used by Sarri et al. ${ }^{6}$. They observed the deflection of protons by magnetic fields at both surfaces of foil targets. In their case, the geometry of sheath electric fields did not affect protons probing the surfaces perpendicularly. The geometry of such fields was known from previous measurements from the same group. In a general case, without such extra knowledge, it may be difficult to discriminate deflections due to magnetic fields from those due to electric fields. In addition, proton deflectrometry results provide only information integrated along the whole proton path.

The change of polarization of a probe beam at normal incidence on a planar foil induced by the Cotton-Mouton effect was used as a diagnostic by Sandhu et al. ${ }^{10}$. In their first experiment, they used a simple photo-diode as detector, thereby without spatial resolution. A following experiment ${ }^{11,12}$ allowed to detect the spatial dependance of the magnetic field. Unfortunately, in this experiment the field of view of the diagnostic was smaller than the actual spatial extension of the magnetic field.

Chatterjee et al. ${ }^{13}$ also measured the change of ellipticity of a probe beam created at the rear side of a $\mathrm{CH}$ target for laser intensity up to $10^{20} \mathrm{~W} / \mathrm{cm}^{2}$. They observed an annular magnetic field up to $50 \mathrm{MG}$ created by the fast electron beam propagating through their target. They also measured the $2 \mathrm{D}$ map of the change of ellipticity of a probe beam at the third harmonic to infer the magnetic turbulence ${ }^{14}$.

A critical point in most of these experiments is that the pre-plasma created by the pump laser pre-pulse was not always experimentally characterized. This is important because the change of polarization depends on both the plasma density and the magnetic field amplitude. Moreover, in analyzing results, the formalism which has normally been applied is valid only for electron density much smaller than the laser probe critical density $\left(n_{e} / n_{c}^{\text {probe }} \ll 1\right)$ and when the electron cyclotron frequency is much smaller than the probe laser frequency $\left(\omega_{c e} / \omega^{\text {probe }} \ll 1\right)$. Such conditions do not always 
hold for a probe beam at normal incidence on target.

In this paper, we present results obtained from a spatially and temporally resolved Cotton-Mouton diagnostic coupled to interferometric measurements. The temporal and spatial evolution of the changes of polarization of the probe laser beam are related to the temporal and spatial evolution of the magnetic fields. Results are compared to PIC simulations allowing a quantitative estimation of the magnetic field. Finally, from our measurements we can estimate the plasma resistivity in presence of a strong magnetic field.

\section{EXPERIMENTAL SET-UP}

In the experiment we used the laser facility ECLIPSE at the CELIA Laboratory, Université de Bordeaux. This is a TiSapphire laser (wavelength $\lambda=800 \mathrm{~nm}$ ) delivering an energy up to $100 \mathrm{~mJ}$ on target within a duration of $27 \mathrm{fs}$ (FWHM). The beam was focused by an off-axis parabola to a minimum size of $8 \mu \mathrm{m}$ (FWHM), with an angle of $45^{\circ}$ with respect to the target normal (p-polarization), yielding a maximum intensity on target of $\approx 10^{18} \mathrm{~W} / \mathrm{cm}^{2}$. The laser intensity contrast was of $10^{-6}$. As targets, we used $0.8 \mu \mathrm{m}$ Al-coated $\mathrm{SiO}_{2}$ glass of optical quality $(\lambda / 5)$. The laser was incident on the Al coated side. Two probe beams were used with $\approx 5 \mathrm{~mJ}$ each, doubled in frequency with BBO (Beta-Barium Borate) crystals. The first probe beam was focused using a $220 \mathrm{~mm}$ focal length lens at normal incidence to obtain a focal spot on target of $\approx 200 \mu \mathrm{m}$. A delay line provided a time delay $\Delta t$ between this probing beam and the main beam within a range of -5 to $150 \mathrm{ps}$ and with time steps of $30 \mathrm{fs}$. The probe beam was reflected at the cut-off density $n_{e}^{c o}$ defined by ${ }^{15}$ :

$$
\frac{n_{e}^{c o}}{n_{c}^{\text {probe }}}+\frac{\omega_{c e}}{\omega^{\text {probe }}}=1 .
$$

This cut-off density is deeper in the target than the critical density for the pump beam $\left(n_{c}^{\text {probe }}=4 n_{c}^{\text {pump }}\right)$, where fast electrons are mainly generated. The reflected probe beam was collected into a polarimeter/imaging system with magnification $\times 10$ and spatial resolution $\approx 12 \mu \mathrm{m}$. Figure 1 shows the setup for the polarimetric measurements.

The second probe beam, used for side-on interferometry measurements of the pre-plasma, allowed us to characterize its density profile which resulted to be approximately exponential. Figure 2 shows the results for the gradient length $L \equiv\left(\nabla n_{e} / n_{e}\right)^{-1}$ recorded 2 ps before the pump laser peak intensity, showing a linear dependence of $L$ on the pre-pulse intensity. 1D simulations performed with the hydrodynamic code $\mathrm{CHIC}^{16}$ reproduced the trend of experimental results. The quantitative difference between experimental and simulations results (a factor 2) is likely due to the fact that the equation of state model used in the simulations was not well adapted to the low-intensity regime typical of laser pedestal. The observed scale lengths $L$ are compatible with electronic temperatures between 40 and $100 \mathrm{eV}$ near the plasma critical density. This estimation directly coming from simulations is also compatible with what can be calculated from $L=c_{s} t$, where $c_{s}$ is the ion-sound velocity in the plasma. a)
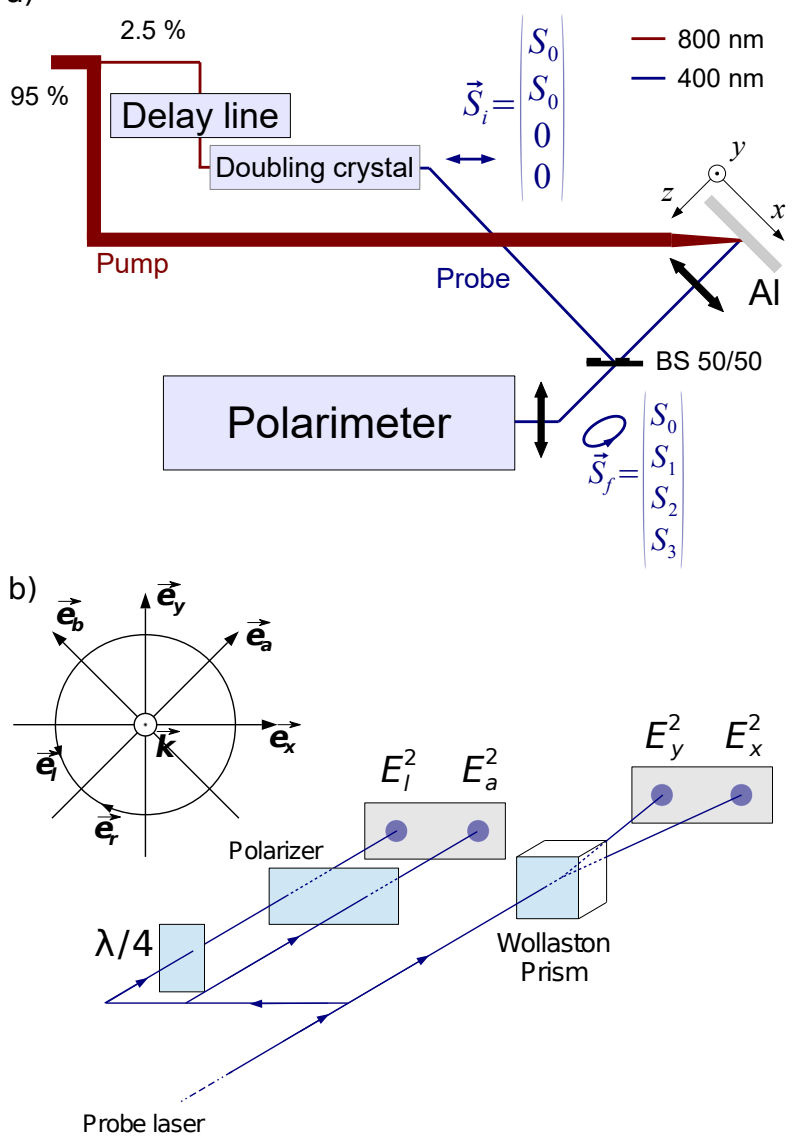

FIG. 1: a) Scheme of the experimental set up showing the pump and the probe laser beams. b) Scheme of the polarimeter. The instrument allows measuring the intensity of the reflected probe laser for four different polarizations: $E_{x}^{2}$, $E_{y}^{2}, E_{a}^{2}$ and $E_{l}^{2} . E_{x}^{2}$ and $E_{y}^{2}$ correspond to linear polarization along two perpendicular axis ( $x$ and $y$ ), $E_{a}^{2}$ corresponds to $45^{\circ}$ rotation and $E_{l}^{2}$ corresponds to (left) circular polarization. The four measurements allow constructing the Stokes vector $\vec{S}$ of the reflected beam and comparing it to the Stokes vector $\overrightarrow{S_{0}}$ of the incoming linearly polarized probe.

The polarimeter is a home-made device that provides four reflectivity images of the target at different polarizations, allowing to obtain the Stokes vector (four Stokes parameters) of the probe beam, related to its polarization ${ }^{17,18}$ :

$$
\vec{S}=\left(\begin{array}{c}
S_{0} \\
S_{0} s_{1} \\
S_{0} s_{2} \\
S_{0} s_{3}
\end{array}\right)=\left(\begin{array}{c}
E_{x}^{2}+E_{y}^{2} \\
E_{x}^{2}-E_{y}^{2} \\
E_{a}^{2}-E_{b}^{2} \\
E_{r}^{2}-E_{l}^{2}
\end{array}\right)
$$

where $E_{x}, E_{y}, E_{a}, E_{b}, E_{r}$ and $E_{l}$ are the electric field projection on the directions defined in figure 1b. This device included two CCDs (one 16 bits and one 12 bits), each one capturing two images, as shown in figure $1 \mathrm{~b}$. The initial probe laser polarization was linear corresponding to the Stokes vector $\overrightarrow{S_{0}}=\left(S_{0}, S_{0}, 0,0\right)$. 


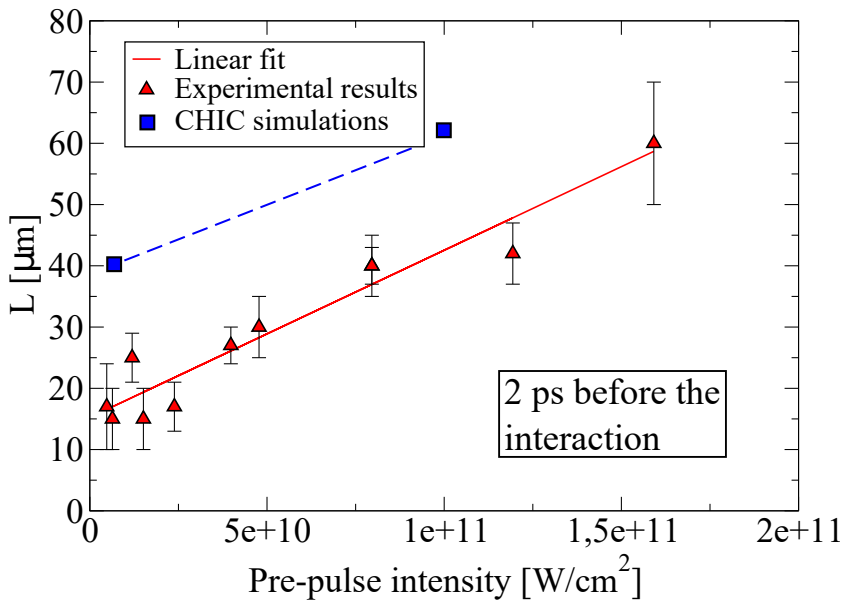

FIG. 2: Plasma density scale-length $L$ at $t=2$ ps before the interaction of the main laser pulse, as a function of pedestal intensity. Triangles: experimental results from interferometry; continuous red line: linear fit of experimental results $L=(2.7 \pm 0.2) \times 10^{-10} I_{\text {pre-pulse }}\left[\mathrm{W} / \mathrm{cm}^{2}\right]+(15 \pm 2)[\mu \mathrm{m}]$.

The maximum intensity of the main pulse used for this measurement was $1.5 \times 10^{17} \mathrm{~W} / \mathrm{cm}^{2}$, corresponding indeed to a maximum pedestal intensity of $1.5 \times 10^{11} \mathrm{~W} / \mathrm{cm}^{2}$. At higher intensity, the second harmonic generation from the interaction of the main pulse with the target was too strong, masking the interferometry probe beam signal.

\section{EXPERIMENTAL RESULTS AND DATA ANALYSIS}

\section{A. Probe beam polarization}

The polarimeter allows to obtain the maps of Stokes parameters of the probe beam after reflection (figure 3 ). In order to superpose the images correctly we calculated the correlation of images at two different polarizations taken on the same object (a laser generated crater). The maximum of this correlation give us the horizontal and vertical shifts needed to superpose the two images. This correlation is performed for all images two-by-two allowing mathematical operations on polarization images and obtaining the Stokes parameters with a precision of $6 \mu \mathrm{m}$. The four images collected on the CCDs also allow detecting the changes of reflectivity of the target following laser irradiation. Figure 3 shows that the size of the region where the reflectivity decreases is smaller than the field of view and much bigger than the focal spot of the pump beam. For an easier visualization of polarization changes, we define two angles, $\chi$ and $\psi$ :

$$
\begin{aligned}
& \chi=\frac{1}{2} \tan ^{-1}\left(\frac{s_{3}}{\sqrt{s_{1}^{2}+s_{2}^{2}}}\right) \\
& \psi=\frac{1}{2} \tan ^{-1}\left(\frac{s_{2}}{s_{1}}\right)
\end{aligned}
$$

representing respectively the ellipticity of the polarization and the orientation of the main polarization axis. These angles allow decoupling the different effects of the magnetic field on

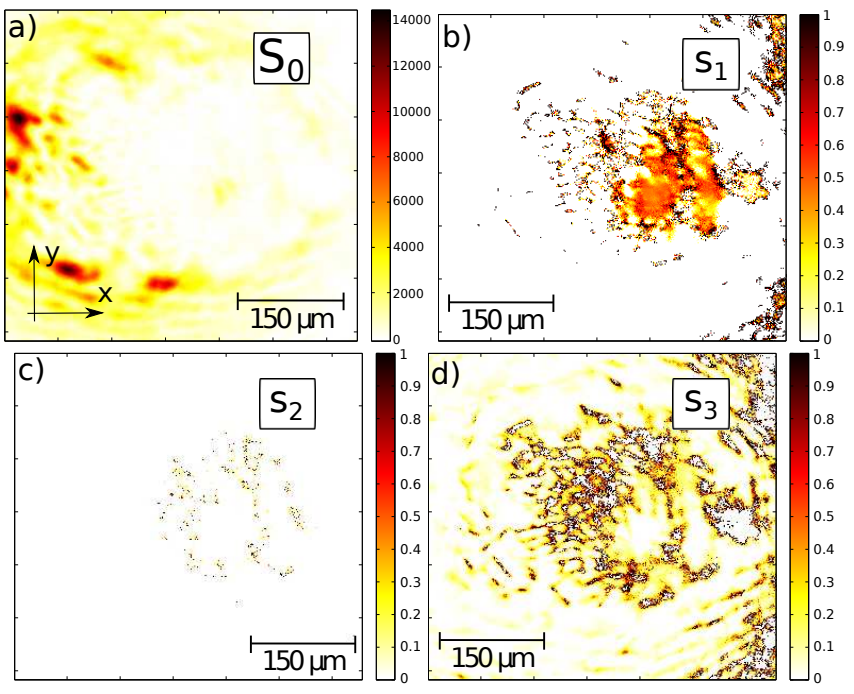

FIG. 3: Spatial mapping of the Stokes parameters of the reflected probe beam $1.5 \mathrm{ps}$ after irradiation by the pump beam. a) $S_{0}$ in color scale. b) $s_{1}=S_{1} / S_{0}$. c) $s_{2}=S_{2} / S_{0}$. d)

$$
s_{3}=S_{3} / S_{0} \text {. }
$$

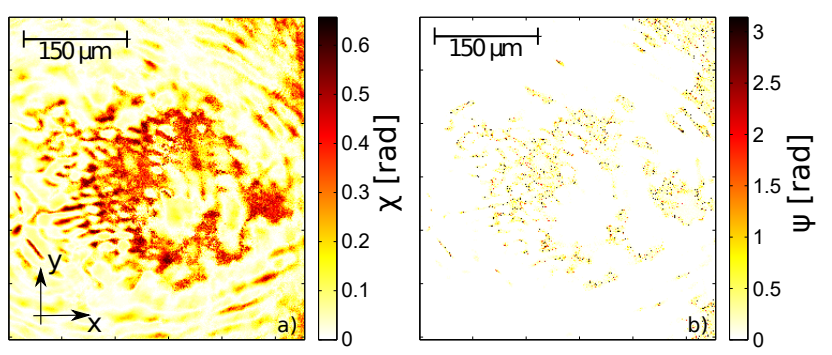

FIG. 4: Spatial mapping of angles $\chi$ (a) and $\psi$ (b) calculated from the Stokes parameters (from figure 3).

polarization. The Faraday effect induces a rotation of polarization (i.e. change of $\psi$ ) if the magnetic field is parallel to the direction of light propagation $\vec{k}$. The Cotton-Mouton effect induces a modification of the polarization ellipticity $\chi$ if the magnetic field is perpendicular to $\vec{k}$. We observe that the region where the reflectivity decreases corresponds to the area where a change of polarization is observed. Because the Cotton-Mouton effect is due to the simultaneous presence of magnetic field and plasma, we see that the radial extension of the plasma (at least a tenuous one) and the extension of the magnetic field are much larger than the size of the focal spot of the pump beam. We observe no appreciable change of the principal direction of polarization (small $\psi$ ) on the back and forth path of the probe beam over the plasma length (figure 4). This implies that we can not infer the longitudinal component of the magnetic field. On the other hand, the change of ellipticity $\chi$ is important near the interaction region, showing a significant Cotton-Mouton effect (magnetic field perpendicular to the target normal). This corresponds to the presence of a strong azimuthal magnetic field in agreement with results al- 
ready presented in the literature. Figure 5a shows the change in time of the ellipticity $\chi$ averaged over the magnetized region. We observe a sharp variation at the time of arrival of the pump pulse on target followed by a slower decay (time scale $\approx 2 \mathrm{ps}$ ). Figure $5 \mathrm{~b}$ shows the change in time of the radius of the magnetized region $\sigma$ (at half-width at half-maximum, HWHM). This increases monotonically in time after the pulse interacts with the target (over our observation time range). The expansion velocity is there $v_{\text {Diff }}=d \sigma / d t \approx 2 \mu \mathrm{m} / \mathrm{ps}$. Figure 5 shows that the ellipticity change and the size $\sigma$ are non-null before 0 ps. This shows the presence of magnetic field before the interaction of the main pulse with the target. Which is likely produced by the laser pedestal, ionizing the target and finally producing a magnetic field by thermoelectric effect.

Figure $5 \mathrm{c}$ shows the variation in time of the size of the magnetised region for different values of the laser intensity. Unlike figure $5 \mathrm{a}$ and $5 \mathrm{~b}$, which were obtained with a good intensity contrast of the laser $\left(\approx 10^{-6}\right)$, these data have been obtained with poorer contrast $\left(\approx 10^{-5}\right)$ hence they cannot be related to data in figure 2 . However, even in these conditions, data show that i) the size of the magnetized region increases with laser intensity, and ii) at zero time there is already a magnetised region with size larger than the focal spot dimension.

In order to extract the quantitative value of the field (from Fig. 5a and 5b), we meet the problem that the relation between ellipticity change and magnetic field value is not bijective. The ellipticity $\chi$ is defined as $\tan \chi=b / a$, with $a$ and $b$ the big and small axis of elliptical polarization, and varies between 0 (for linear polarization $b=0$ ) and $\pi / 4$ (for circular polarization $a=b$ )(equation 3 ). When the magnetic field is strong enough, the big axis $a$ and small axis $b$ reverse changing the polarization from circular polarization to linear (passing from linear to circular and then to linear again). This effect can be evidenced by explicitly solving the equations of the propagation of an electro-magnetic wave inside a magnetized plasma. Figure 6 shows the change in ellipticity after a back and forth path of an electromagnetic wave inside of a plasma with exponential profile, for different values of the characteristic gradient length $L$ and of the magnetic field (azimuthal amplitude). We see that a measured ellipticity change can correspond to different values of magnetic field. We notice that when $n_{e} \ll n_{c}$ and $\omega_{c e} \ll \omega$, this ambiguity is removed and a bijective relation between ellipticity and magnetic field strength is obtained as it was assumed in ${ }^{10}$ and $^{11}$.

If the temporal variation of $\chi$ is slow, we can apply a continuity hypothesis and extract magnetic field values (for the measured density profiles) at a given time knowing the field at earlier times. This is certainly true for the slow decay observed in figure 5 for $\Delta t>0$. However at earlier times (i.e. at the beginning of the interaction) there is a quick jump of magnetic field from zero to a maximum value which cannot be extracted from experimental data only. For this reason, we needed to complement our measurements with results from 2D PIC simulations (figure 8) performed with the code PI$\mathrm{CLS}^{19}$.
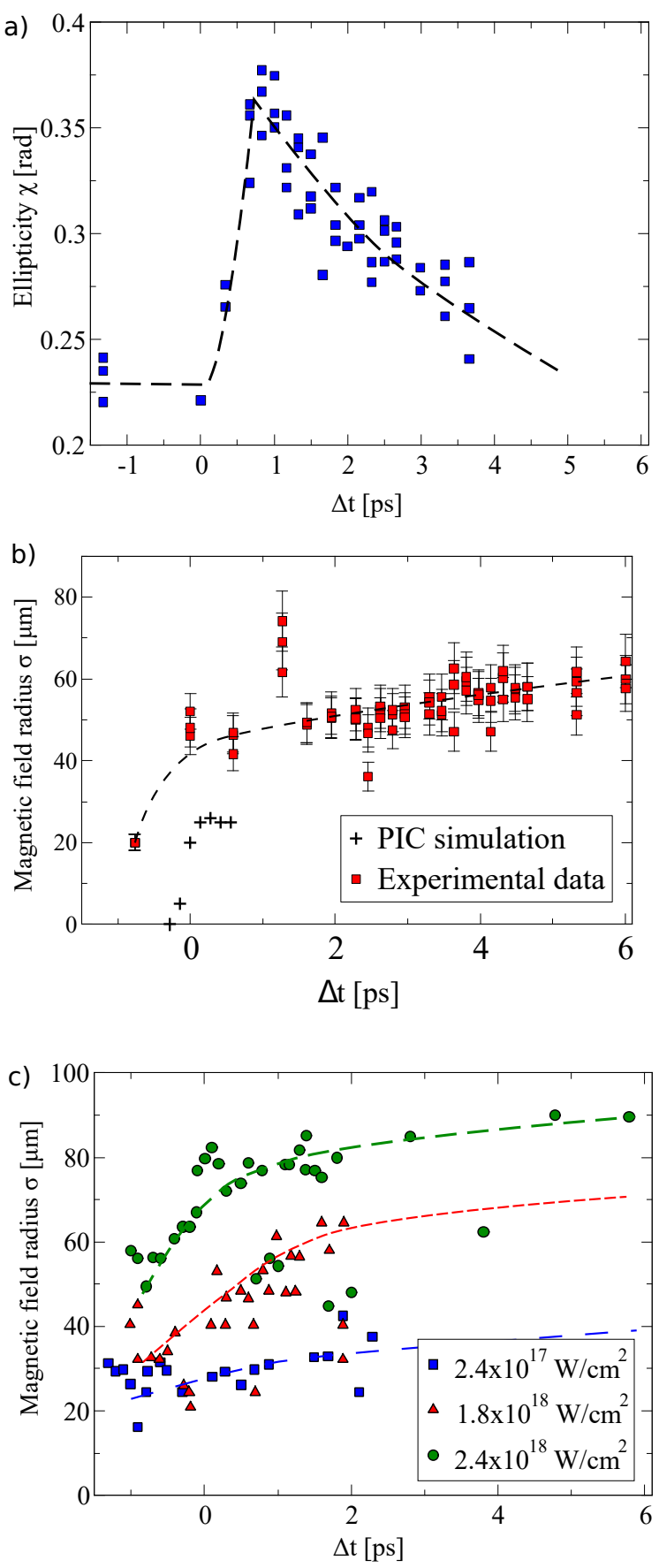

FIG. 5: a) Spatially-averaged change of ellipticity versus time obtained during the interaction of a $4 \times 10^{17} \mathrm{~W} / \mathrm{cm}^{2}$ laser $\left(10^{-6}\right.$ intensity contrast) with an $\mathrm{Al}$ coated target. b) Corresponding evolution of the radius of the magnetized region $\sigma$ (at HWHM) with time. The evolution size of the PIC simulation is also shown. c) Evolution of $\sigma$ for three laser intensities at low contrast, $10^{-5}$. To clarify visualisation, trend lines are plotted on the graphs. 


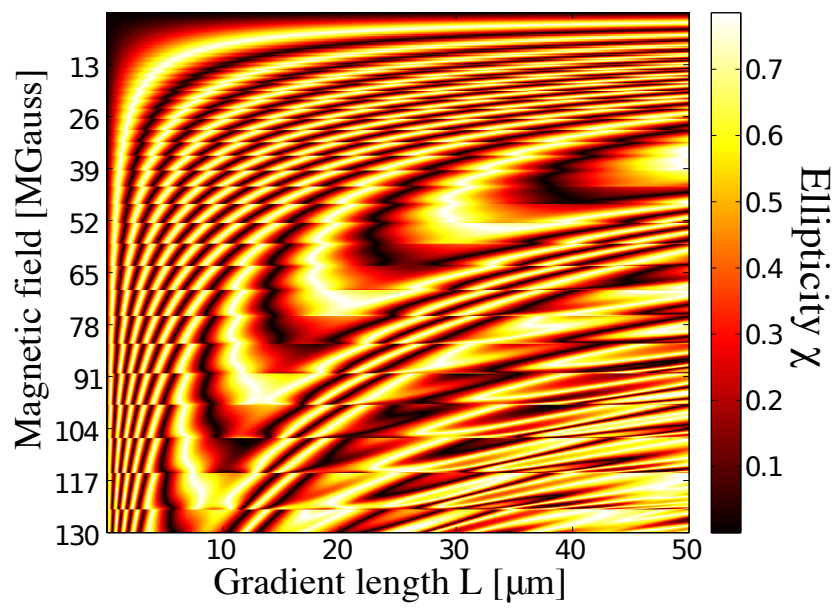

FIG. 6: Calculated change in polarization ellipticity after the back and forth path in a non-uniform plasma with an exponential density profile of gradient length $L$ and a constant magnetic field. Results are obtained by solving the equations for the propagation of an electromagnetic wave inside of a magnetized plasma ${ }^{17}$.

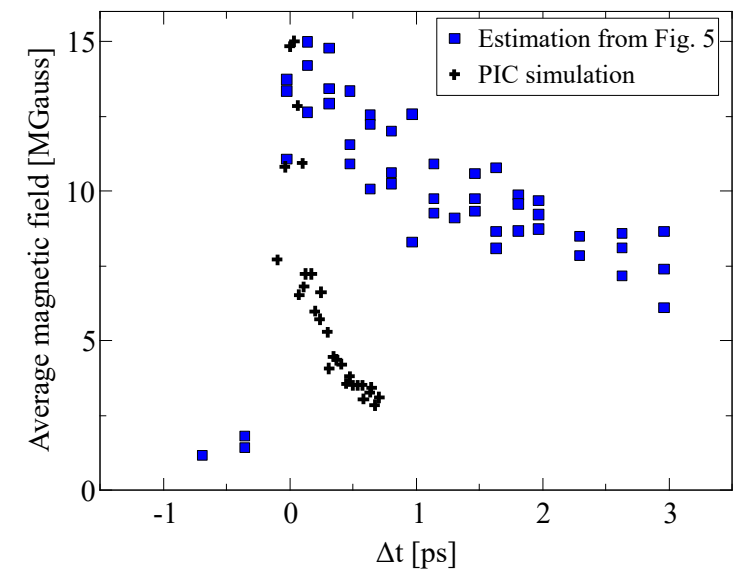

FIG. 7: Square: average magnetic field obtained from the ellipticity variation (figure 5a). The maximum magnetic field amplitude has been matched to PIC simulation results.

Crosses: The temporal evolution of the average magnetic field obtained from PIC simulation.

\section{B. PIC simulation}

The code used a simulation box of $60 \times 20 \lambda$ with a resolution of $\lambda / 25$ in order to simulate the interaction of a laser at $4 \times 10^{17} \mathrm{~W} / \mathrm{cm}^{2}$ with a plasma of aluminum, with a preplasma characterized by an exponential profile (the gradient length is consistent with the experimental data shown in figure 2). A strong and filamented magnetic field is developing near the critical density (figure $8 \mathrm{a}$ ) over $\mathrm{a} \approx 50 \mu \mathrm{m}$ region. It reaches a maximum of $\approx 15 \mathrm{MG}$ at $\approx 150 \mathrm{fs}$ and then decreases slowly, with a characteristic time of $150 \mathrm{fs}$, down to 1.3 MG (crosses in figure 7). The peak of magnetic field is
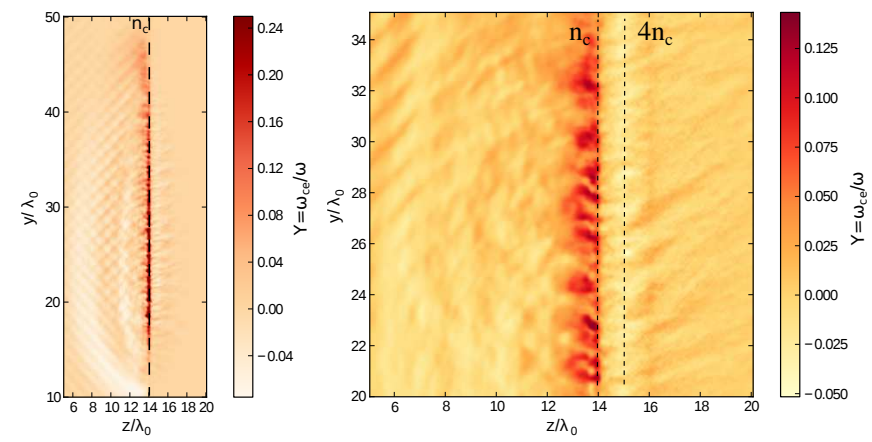

FIG. 8: a) PIC magnetic field map for the interaction of a $4 \times 10^{17} \mathrm{~W} / \mathrm{cm}^{2}$ laser with a $1 \mu \mathrm{m} \mathrm{Al}$ coating on a $\mathrm{CH}$ target, after $140 \mathrm{fs}$. b) Zoom of the interaction region.

created by the propagation of fast electrons near the critical density (figure 8b).

The characteristic filament sizes are 1 to $2 \mu \mathrm{m}$ and hence cannot be resolved by our diagnostic which has a spatial resolution $\gtrsim 10 \mu \mathrm{m}$. Using PIC results to estimate the value of the averaged magnetic field over the filament size at early times we retrieve the graph of figure 7 . We notice that the PIC simulations underestimate the size of the magnetized region by a factor $\simeq 2$ (figure $5 \mathrm{~b}$ ). This is likely due to the presence of wings in the intensity distribution in the experimental focal spot, which could contain a non-negligible energy, and whose presence is not taken into account in PIC simulations. We also notice the different decay time of the magnetic field (figure 7). This difference comes from the incapacity of the PIC code to correctly describe the plasma resistivity created by the contrapropagating electrons inside the plasma.

\section{Plasma resistivity}

The spatial and temporal evolutions of the magnetic field (figure 5b) allow to retrieve the resistivity $\eta$ of the plasma. In order to do an explicit analytical calculation we used here the ansatz $\vec{B}=\vec{B}_{0} \exp \left(-t / \tau_{0}-r^{2} /\left(2 \sigma^{2}\right)\right)$ [this corresponds to spatially averaging the many short-wavelength fluctuations of the magnetic field which are evident in the results of figure 4]. With this assumption we have solved the differential equation for the resistive diffusion of the magnetic field $\frac{\partial \vec{B}}{\partial t}=\frac{\eta}{\mu_{0}} \triangle \vec{B}$. The calculation brings to $\eta \approx \mu_{0}\left(\sigma^{2} / \tau_{0}\right) \approx \mu_{0} \sigma \frac{d \sigma}{d t}$. Here the diffusion velocity $v_{\text {Diff }}=\frac{d \sigma}{d t}$ is the slope determined from figure $5 \mathrm{~b}$. This yields a resistivity $\eta=(6 \pm 3.5) \times 10^{-5} \Omega . \mathrm{m}$. An even higher resistivity is obtained by inserting the decay time obtained from figure $5 \mathrm{a}$.

The method gives an average resistivity higher than what is expected in a non-magnetized plasma, of $\eta_{t h}=5 \times 10^{-6} \Omega$.m according to the Eidmann-Huller-Chimmier model ${ }^{20,21}$ in the conditions of density (between $n_{c}$ and $4 n_{c}$, maximum penetration of the probe beam) and plasma temperature inferred from interferometry measurements and hydrodynamic simulations (see figure 9).

The difference should be inferred from the presence of 


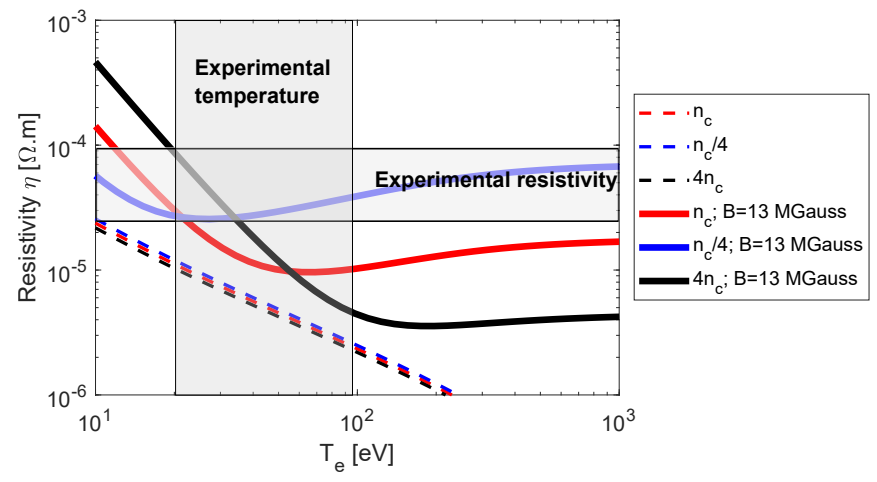

FIG. 9: Plasma resistivity from Eidmann-Huller-Chimier's model (dashed curves) and the correction induced by the presence of a (13 MG) magnetic field inducing an anisotropy (full curves), for three different densities: $n_{c} / 4, n_{c}$ and $4 n_{c}$.

The vertical and horizontal bars identify the estimated experimental ranges of, respectively, temperature and resistivity.

magnetic field and its influence on the collision frequency. This creates an anisotropy in the resistivity tensor as ${ }^{22}$ :

$$
\eta=\left(\begin{array}{ccc}
\eta_{\perp} & \eta_{\wedge} & 0 \\
-\eta_{\wedge} & \eta_{\perp} & 0 \\
0 & 0 & \eta_{\|}
\end{array}\right),
$$

where $\|$ indicates the direction parallel to $B, \perp$ the direction perpendicular to $B$ and parallel to $E$ and $\wedge$ the direction perpendicular to $B$ and perpendicular to $E$. In our case this leads to an increase of the resistivity $\eta_{\perp}$, as ${ }^{23}$ :

$$
\eta_{\perp}=\eta \frac{1+\omega_{c}^{2} \tau^{2}}{\omega_{c} \tau}
$$

with $\tau$ is the inverse of the collision frequency described by Eidmann et al. ${ }^{21}$. Since our estimation of the resistivity is based on the variation of the magnetic-field radial extension $\sigma$, indeed we are measuring the perpendicular component of resistivity $\eta_{\perp}$, the magnetic field being azymuthal. By assuming a magnetic field of $13 \mathrm{MG}$ (from figure 7) the theoretical resistivity changes from $\eta_{\perp}=5 \times 10^{-6} \Omega$.m to $\eta_{\perp}=5 \times 10^{-5} \Omega$.m, which is consistent with our estimation from experimental results (figure 9). Let us notice that the temperature inferred from the interferometer measurement and hydrodynamic simulations (Figure 2) concerns the preplasma and can therefore be smaller than the actual temperature after the interaction of the main beam. On the other side, in our model, we have taken into account only the surface diffusion of the magnetic field. In reality the magnetic field will also diffuse inside the target, i.e. into layers characterized by bigger densities and lower temperatures. In any case, Fig. 9 shows that, even extending the range of densities and temperatures, the experimentally measured resistivity clearly indicates plasma magnetization, as it does not match the EidemannHuller-Chimier prediction for a non-magnetized plasma by about one order of magnitude.

\section{CONCLUSIONS}

To conclude, the optical polarimetry diagnostic used in this experiment is suited for measuring the evolution of plasma magnetic fields parallel to a foil-target surface generated during the interaction of a high intensity laser. Our measurements allow to characterize the temporal and spatial evolution of the magnetic fields, and from this estimate the induced anisotropy on the plasma resistivity due to the strong magnetization.Let us notice that a resistivity higher than expected for a non magnetized plasma was also observed in experimental conditions similar to ours in the works in references ${ }^{10}$ and ${ }^{12}$. In order to explain such results, the authors speculated that it could be due to Electro-Magnetic Hydrodynamic Dynamic Turbulence (which could not be characterised in their experiment). On the basis of our measurements and conclusions, we believe instead that a simpler explanation of the high resistivity is simply due to the presence of the magnetic field.

\section{ACKNOWLEDGMENTS}

We are grateful to Christopher Spindloe from Scitech for producing the targets used in the experiment. This work was performed through funding from the French National Agency for Research (ANR) and the competitiveness cluster Alpha-Route des Lasers, project number TERRE ANR-2011BS04-014, and within the framework of the 'Investments for the future' program IdEx Bordeaux LAPHIA (ANR-10IDEX-03-02). The research has also been carried out in the framework of the EUROfusion Consortium and has received funding from the Euratom research and training programme 2014-2018 under grant agreement No. 633053. The views and opinions expressed herein do not necessarily reflect those of the European Commission. The work was also partially supported by the Competitiveness Program of NRNU MEPhI, Russia.

\footnotetext{
${ }^{1}$ M. G. Haines, "Magnetic-field generation in laser fusion and hot-electron transport," Canadian Journal of Physics 64, 912 (1986).

${ }^{2}$ F. Pérez, A. J. Kemp, L. Divol, C. D. Chen, and P. K. Patel, "Deflection of mev electrons by self-generated magnetic fields in intense laser-solid interactions," Phys. Rev. Lett. 111, 245001 (2013).

${ }^{3}$ J. Adam, A. Héron, and G. Laval, "Dispersion and transport of energetic particules due to the interaction of intense laser pulses with overdense plasmas," Physical Review Letters 97 (2006).

${ }^{4}$ M. Borghesi, A. J. Mackinnon, R. Gaillard, O. Willi, A. Pukhov, and J. Meyer-ter Vehn, "Large quasistatic magnetic fields generated by a relativistically intense laser pulse propagating in a preionized plasma," Phys. Rev. Lett. 80, 5137-5140 (1998).

${ }^{5}$ M. Tatarakis, A. Gopal, I. Watts, F. N. Beg, A. E. Dangor, K. Krushelnick, U. Wagner, P. A. Norreys, E. L. Clark, M. Zepf, and R. G. Evans, "Measurements of ultrastrong magnetic fields during relativistic laser-plasma interactions," Physics of Plasmas 9, 2244-2250 (2002).

${ }^{6}$ G. Sarri, A. Macchi, C. A. Cecchetti, S. Kar, T. V. Liseykina, X. H. Yang, M. E. Dieckmann, J. Fuchs, M. Galimberti, L. A. Gizzi, R. Jung, I. Kourakis, J. Osterholz, F. Pegoraro, A. P. L. Robinson, L. Romagnani, O. Willi, and M. Borghesi, "Dynamics of self-generated, large amplitude magnetic fields following high-intensity laser matter interaction," Phys. Rev. Lett. 109, 205002 (2012).

${ }^{7}$ P. Gibbon, Short Pulse Laser Interactions with Matter: An Introduction (Imperial College Press, 2005).
} 
${ }^{8}$ T. Pisarczyk, S. Y. Gus'kov, T. Chodukowski, R. Dudzak, P. Korneev, N. N. Demchenko, Z. Kalinowska, J. Dostal, A. Zaras-Szydlowska, S. Borodziuk, L. Juha, J. Cikhardt, J. Krasa, D. Klir, B. Cikhardtova, P. Kubes, E. Krousky, M. Krus, J. Ullschmied, K. Jungwirth, J. Hrebicek, T. Medrik, J. Golasowski, M. Pfeifer, O. Renner, S. Singh, S. Kar, H. Ahmed, J. Skala, and P. Pisarczyk, "Kinetic magnetization by fast electrons in laser-produced plasmas at sub-relativistic intensities," Physics of Plasmas 24, 102711 (2017).

${ }^{9}$ A. Gopal, S. Minardi, M. Burza, G. Genoud, I. Tzianaki, A. Karmakar, P. Gibbon, M. Tatarakis, A. Persson, and C.-G. Wahlström, "Megagauss magnetic field generation by ultra-short pulses at relativistic intensities," Plasma Physics and Controlled Fusion 55, 035002 (2013).

${ }^{10}$ A. S. Sandhu, A. K. Dharmadhikari, P. P. Rajeev, G. R. Kumar, S. Sengupta, A. Das, and P. K. Kaw, "Laser-generated ultrashort multimegagauss magnetic pulses in plasmas," Phys. Rev. Lett. 89, 225002 (2002).

${ }^{11}$ S. Mondal, V. Narayanan, A. D. Lad, S. Ahmed, S. Sengupta, A. Das, Z. M. Sheng, P. K. Kaw, and G. R. Kumar, "Measurement of hot electron transport in overdense plasma via self induced giant magnetic pulses," Journal of Physics: Conference Series 244, 022049 (2010).

${ }^{12}$ S. Kahaly, S. Mondal, G. R. Kumar, S. Sengupta, A. Das, and P. K. Kaw, "Polarimetric detection of laser induced ultrashort magnetic pulses in overdense plasma," Physics of Plasmas 16, 043114 (2009).

${ }^{13}$ G. Chatterjee, P. K. Singh, A. P. L. Robinson, D. Blackman, N. Booth, O. Culfa, R. J. Dance, L. A. Gizzi, R. J. Gray, J. S. Green, P. Koester, G. R. Kumar, L. Labate, A. D. Lad, K. L. Lancaster, J. Pasley, N. C. Woolsey, and P. P. Rajeev, "Micron-scale mapping of megagauss magnetic fields using optical polarimetry to probe hot electron transport in petawatt-class lasersolid interactions,” Scientific Reports 7, 8347 (2017).
${ }^{14}$ G. Chatterjee, K. Schoeffler, P. Singh, A. Adak, A. Lad, S. Sengupta, P. Kaw, L. O. Silva, A. Das, and G. R. Kumar, "Magnetic turbulence in a table-top laser-plasma relevant to astrophysical scenarios," Nature Communications 8, 15970 (2017).

${ }^{15} \mathrm{M}$. Heald and C. Wharton, Plasma diagnostics with microwaves (John Wiley \& Sons Inc., 1965).

${ }^{16} \mathrm{~J}$. Breil, S. Galera, and P. H. Maire, "Multi-material ALE computation in inertial confinement fusion code CHIC," Computers \& Fluids 46, 161-167 (2011).

${ }^{17}$ S. E. Segre, "A review of plasma polarimetry - theory and methods," Plasma Physics and Controlled Fusion 41, R57 (1999).

${ }^{18}$ W. H. McMaster, "Polarization and the stokes parameters," American Journal of Physics 22, 351-362 (1954).

${ }^{19}$ Y. Sentoku and A. Kemp, "Numerical methods for particle simulations at extreme densities and temperatures: Weighted particles, relativistic collisions and reduced currents," Journal of Computational Physics 227, 6846 6861 (2008)

${ }^{20}$ B. Chimier, V. T. Tikhonchuk, and L. Hallo, "Heating model for metals irradiated by a subpicosecond laser pulse," Phys. Rev. B 75, 195124 (2007).

${ }^{21}$ K. Eidmann, J. Meyer-ter Vehn, T. Schlegel, and S. Hüller, "Hydrodynamic simulation of subpicosecond laser interaction with solid-density matter," Physical Review E - Statistical Physics, Plasmas, Fluids, and Related Interdisciplinary Topics 62, 1202-1214 (2000).

${ }^{22}$ W. Stygar, G. Gerdin, and D. L Fehl, "Analytic electrical-conductivity tensor of a nondegenerate lorentz plasma," Physical review. E, Statistical, nonlinear, and soft matter physics 66, 046417 (2002).

${ }^{23} \mathrm{~B}$. Smirnov, Introduction to plasma physics (MIR Publishers Moscow, 1977). 
a)

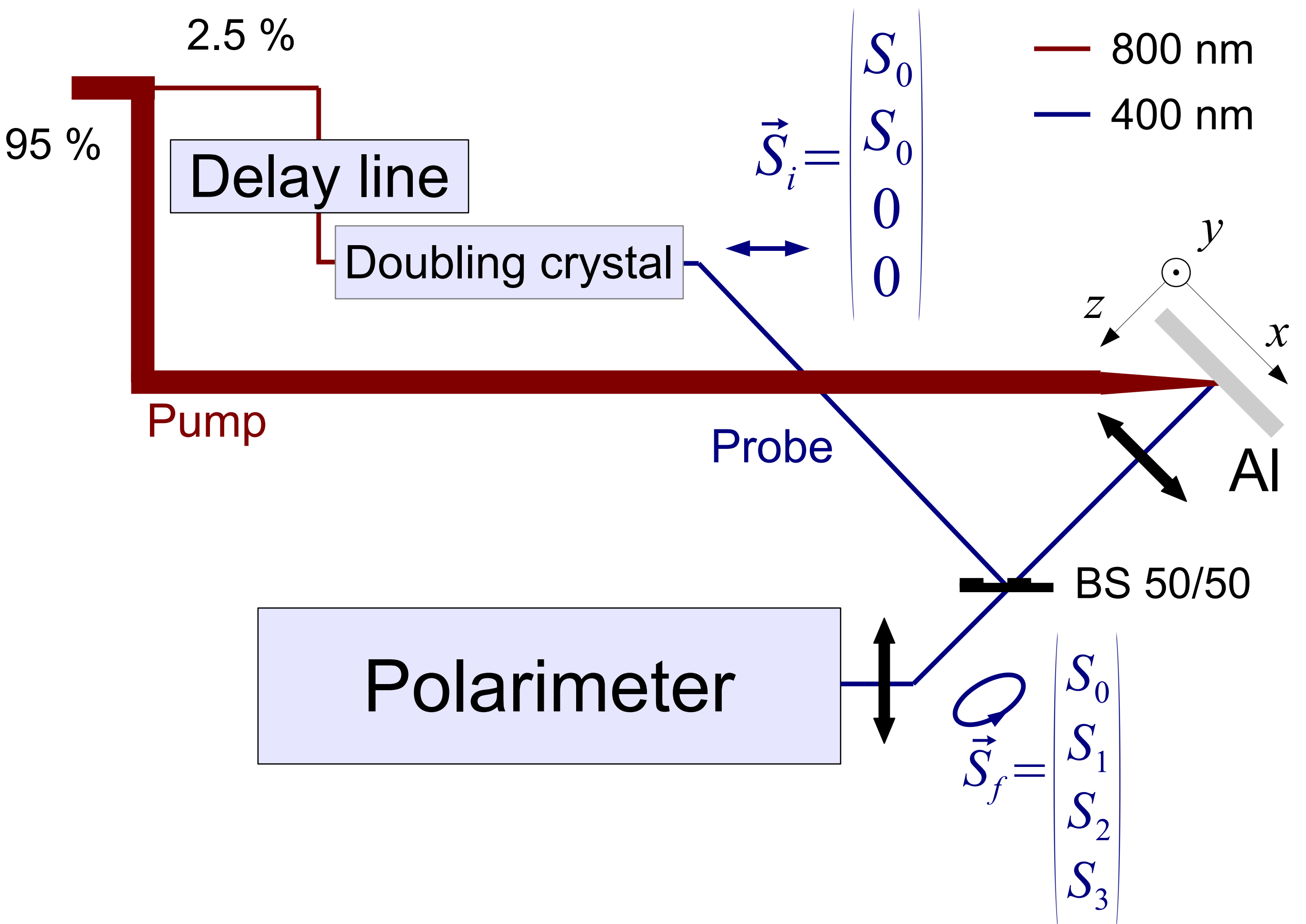




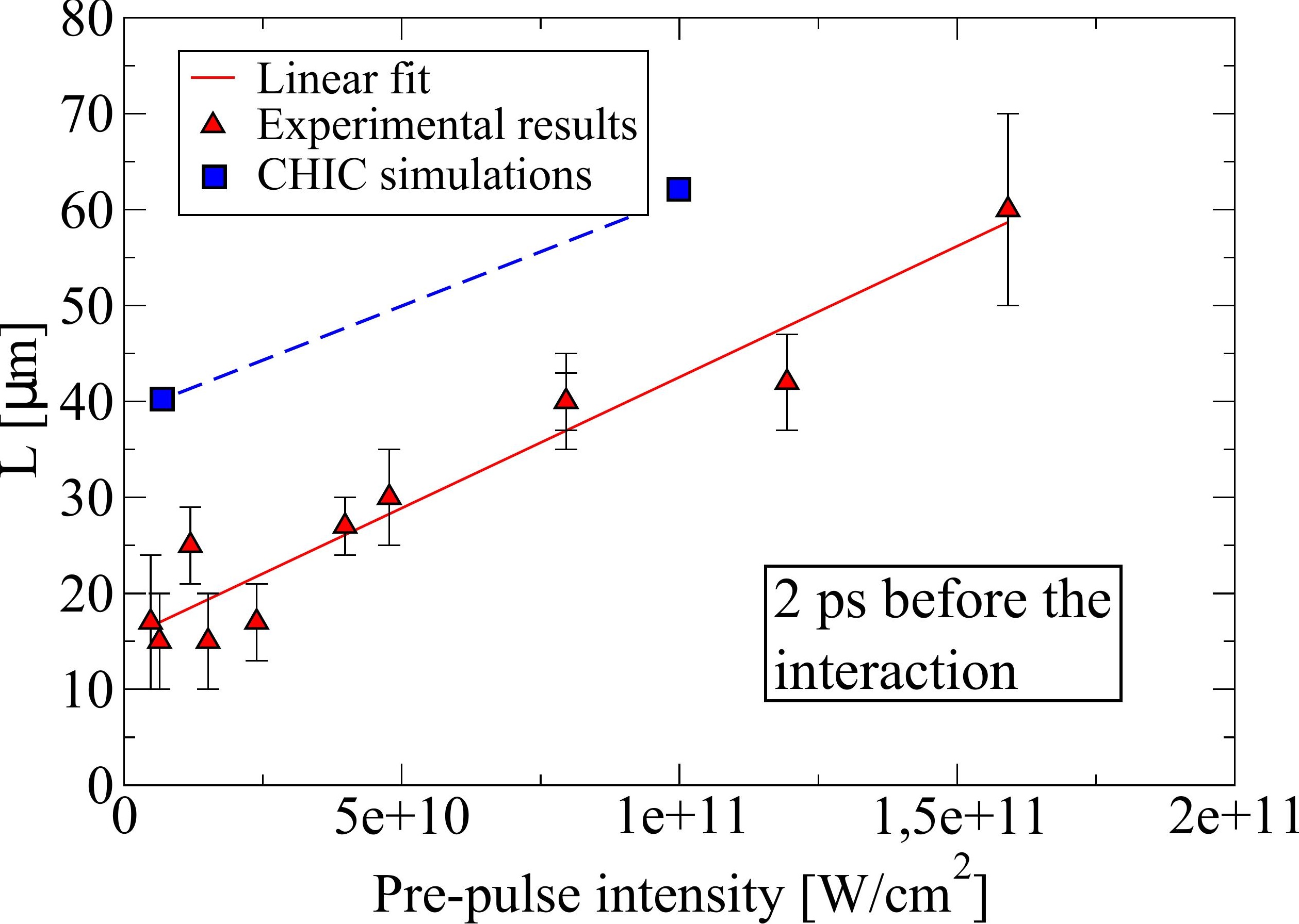




\section{a)}

14000

12000

10000

8000

6000

4000

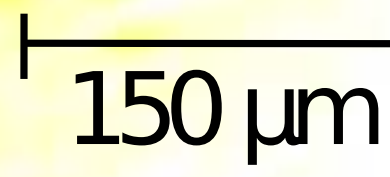

$-2000$ 


\section{b)}

"अ

$2 \sin ^{\circ}$ '* $8 \mathbf{s i}$.

ᄀ.

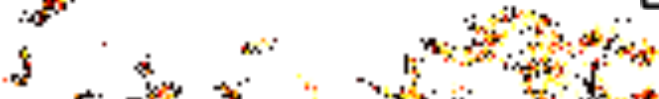

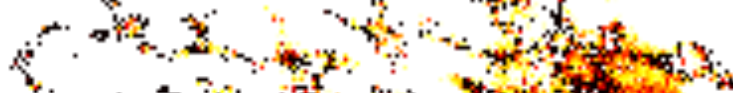

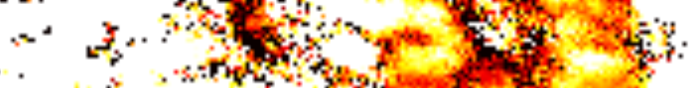

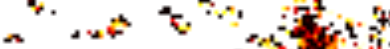

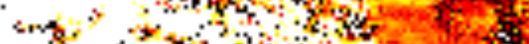

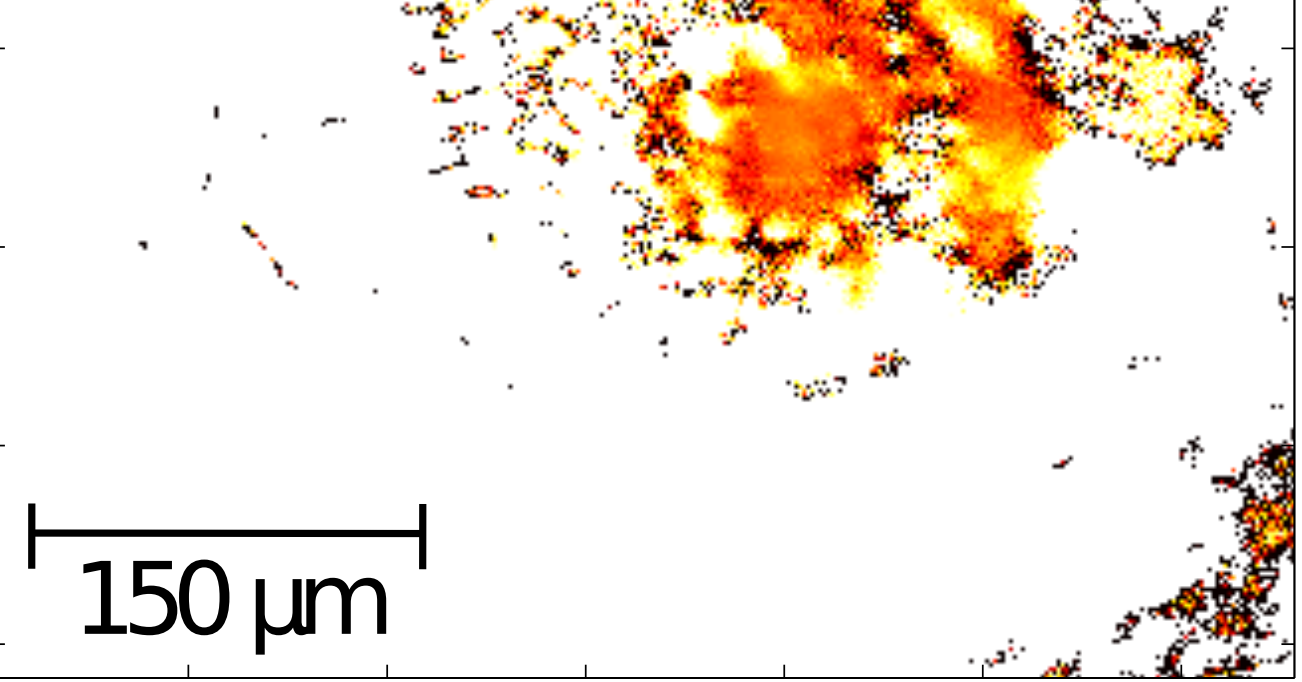
0.5
0.4
0.3
0.2
0.1
0 


\section{C)}

\section{$\mathrm{S}_{2}$}

0.9

0.8

0.7

0.6

0.5

0.4

0.3

0.2

$\longmapsto$

0.1 


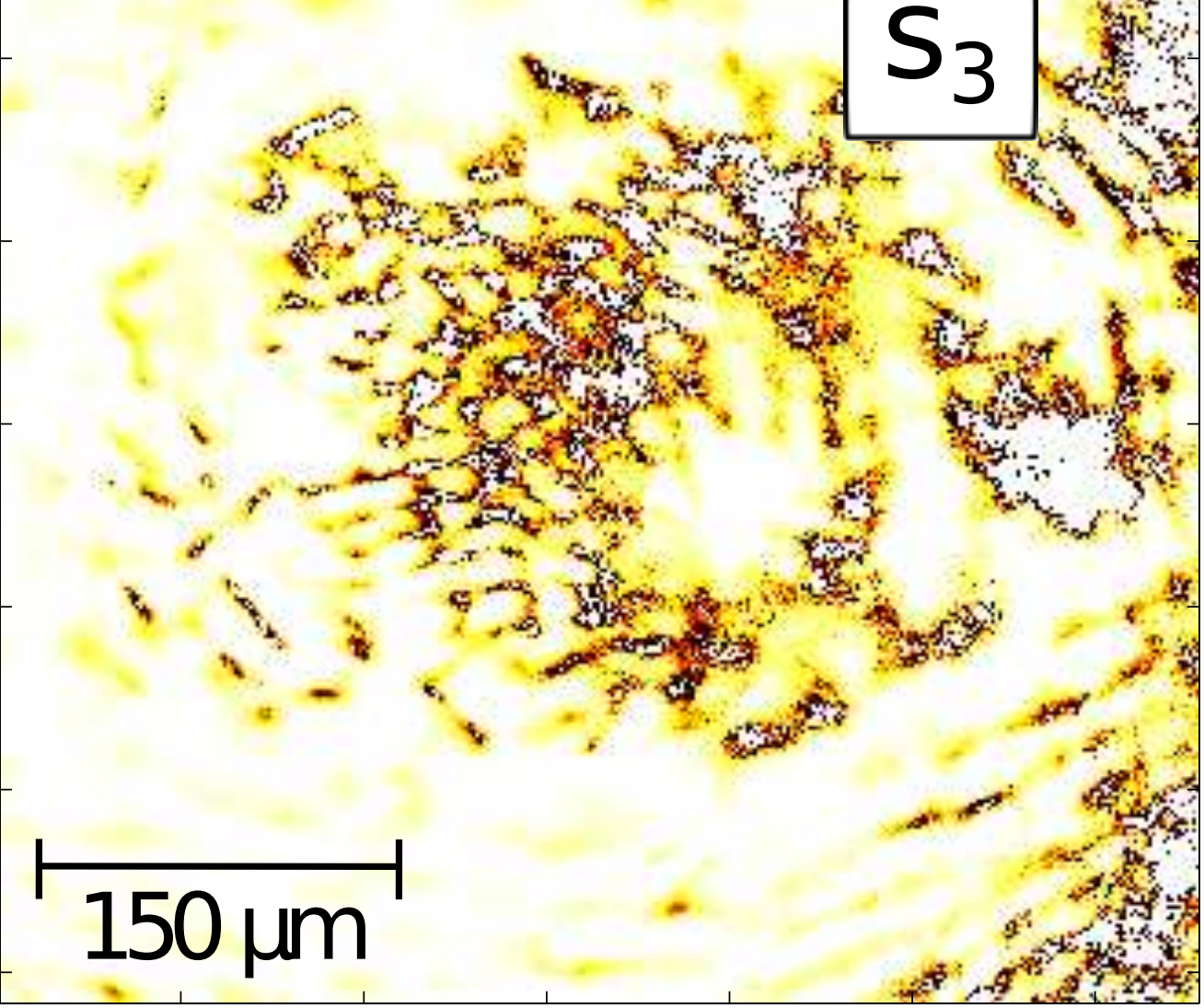

0.9

0.8

0.7

0.6

0.5

0.4

0.3

0.2

0.1

0 


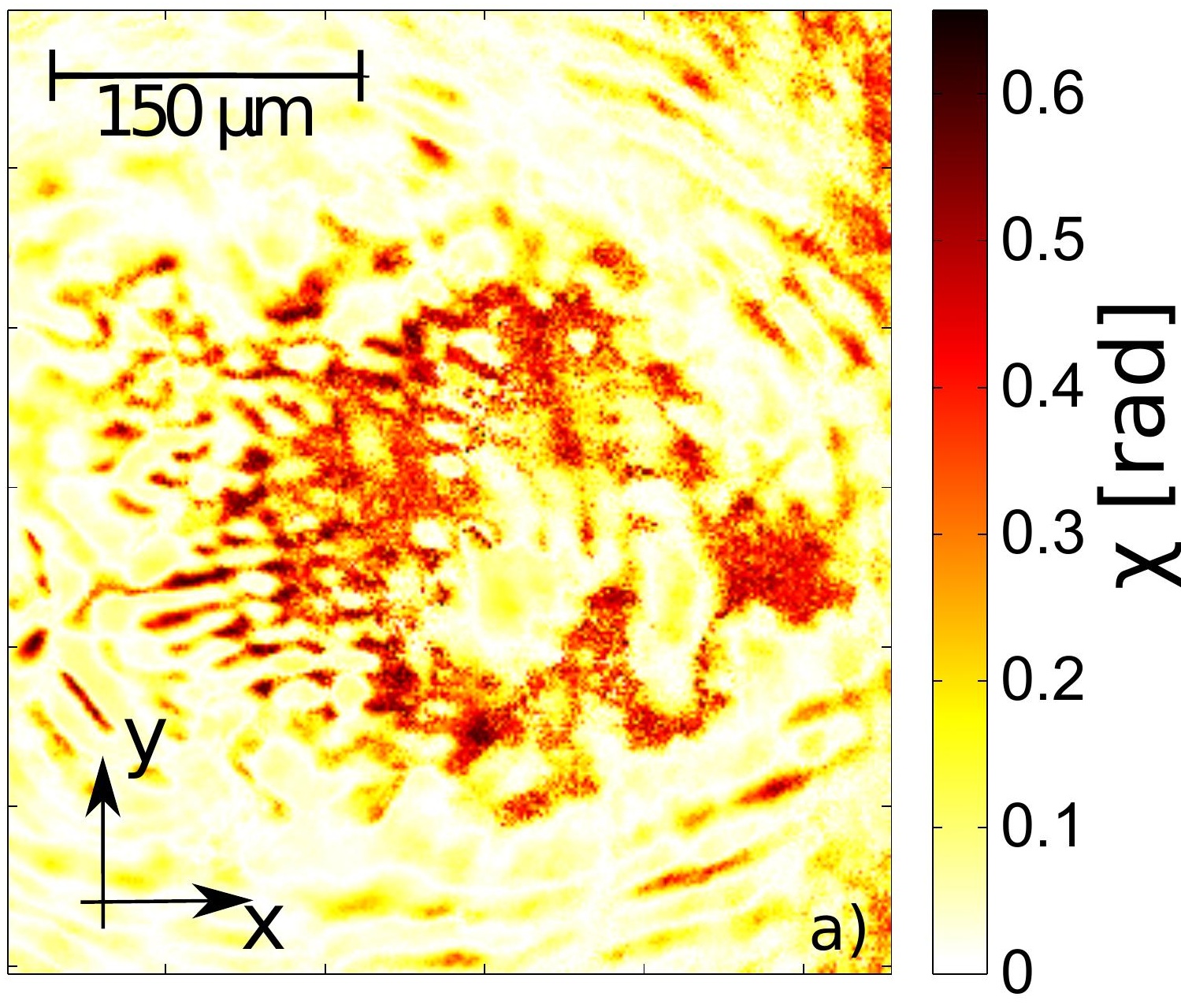




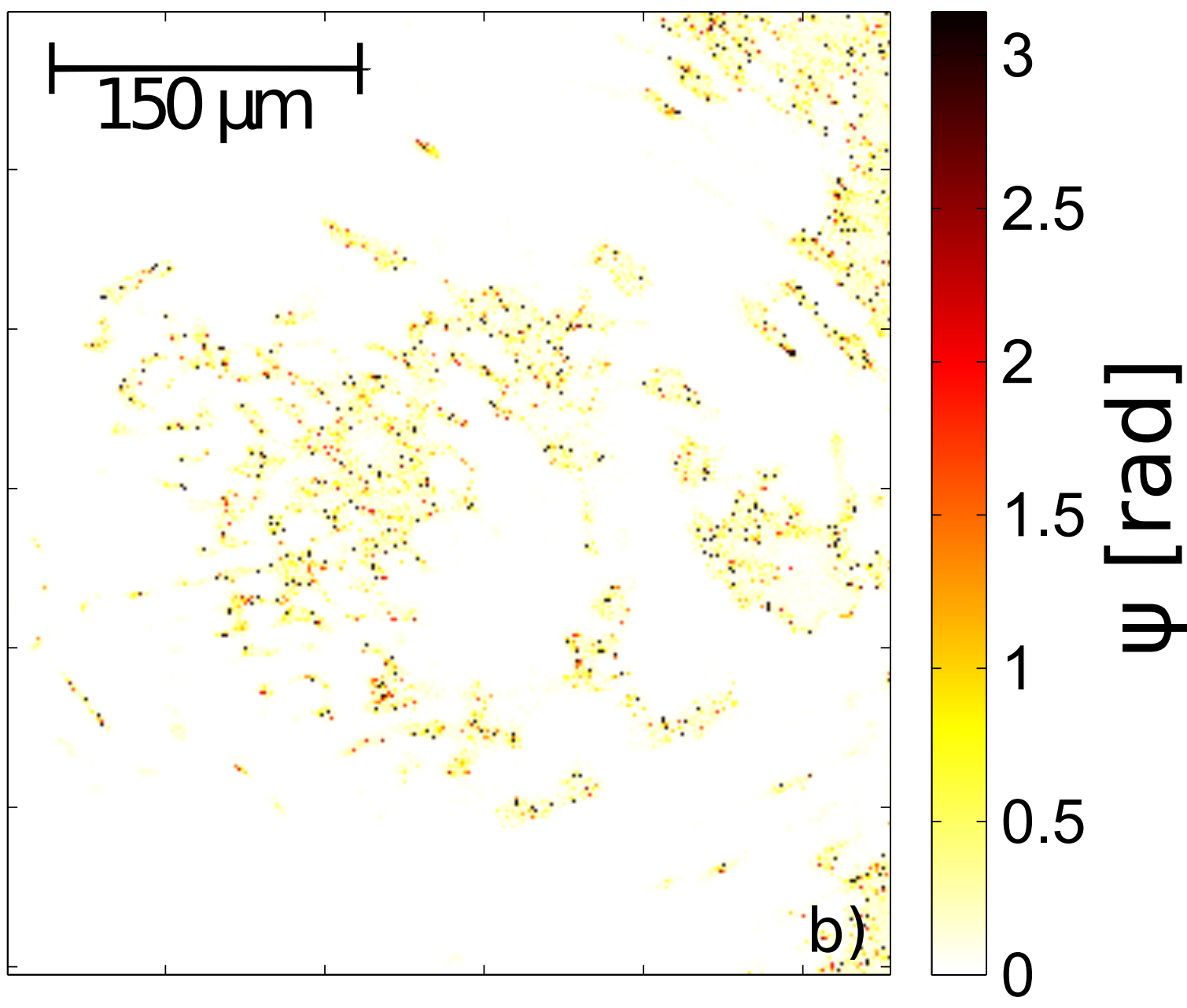




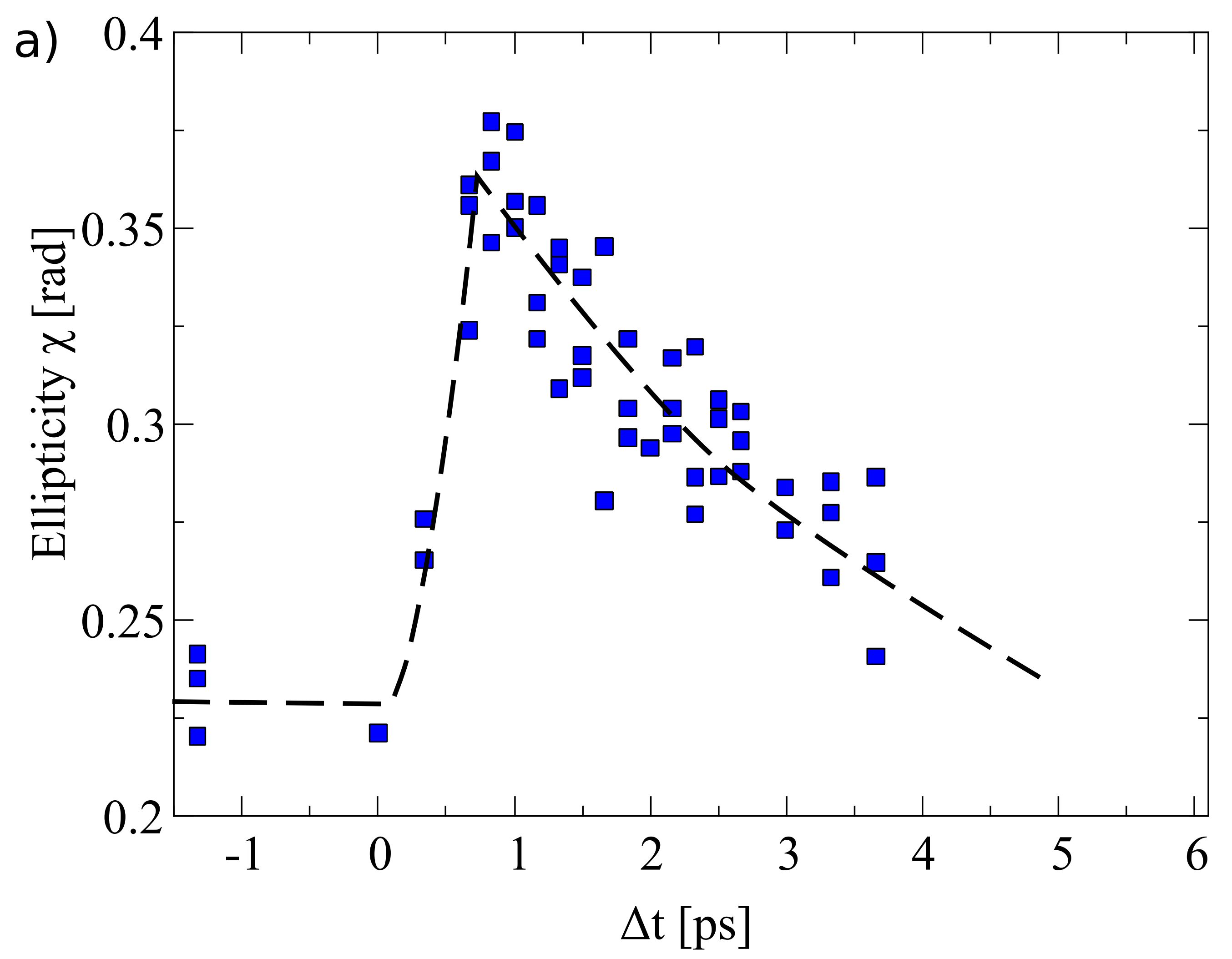




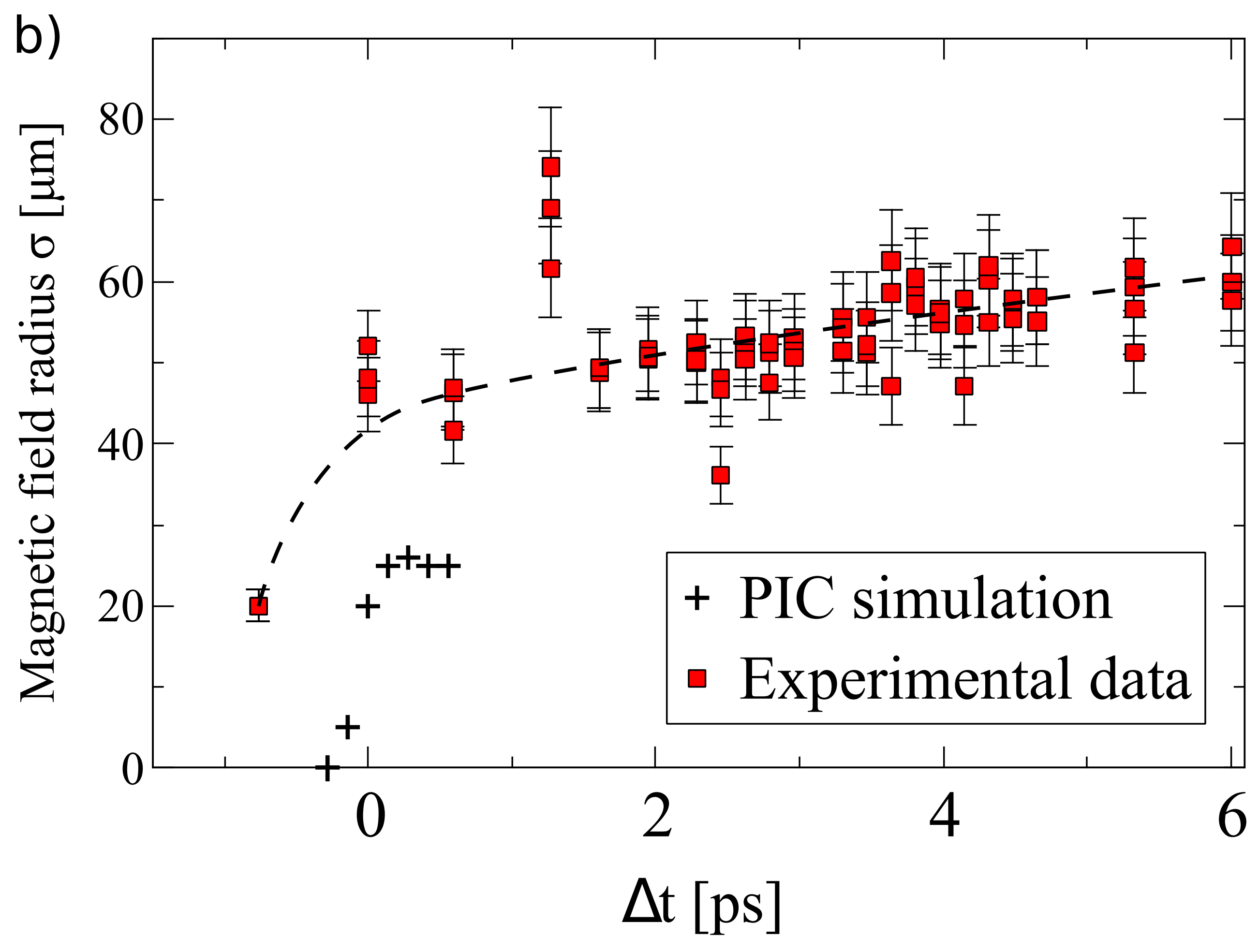




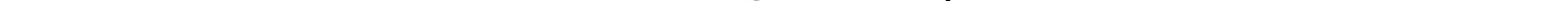




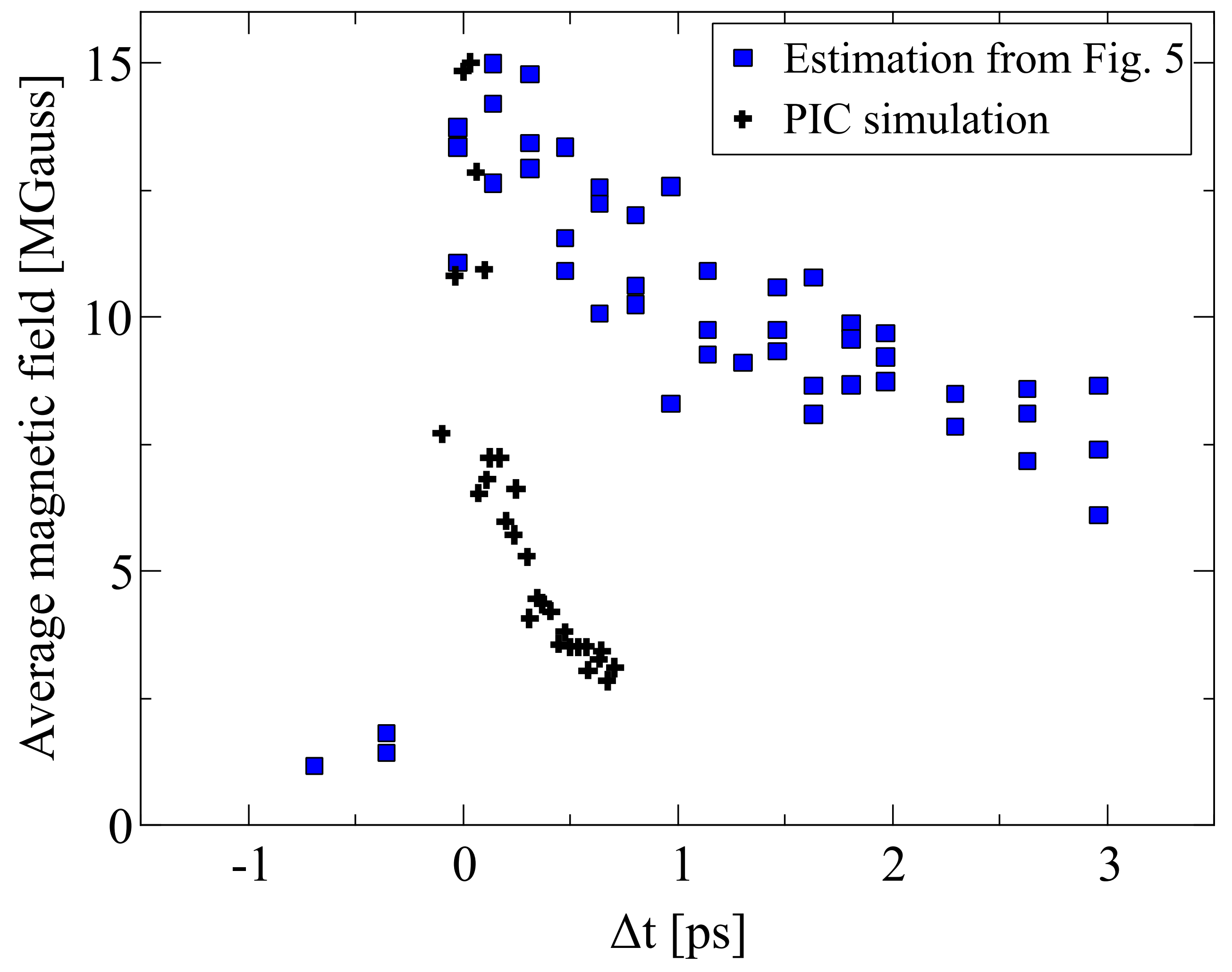




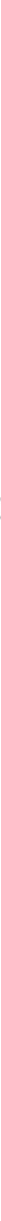




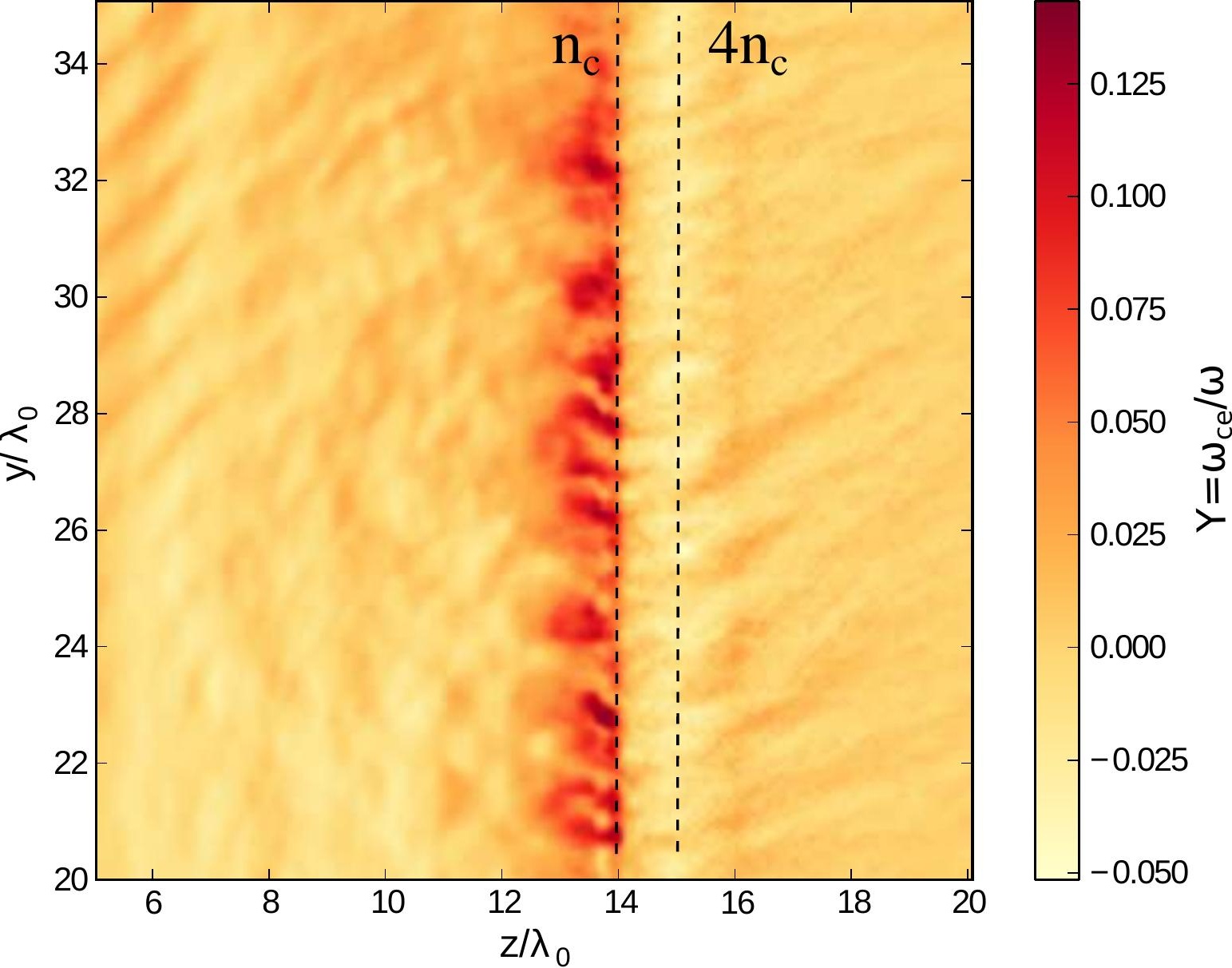




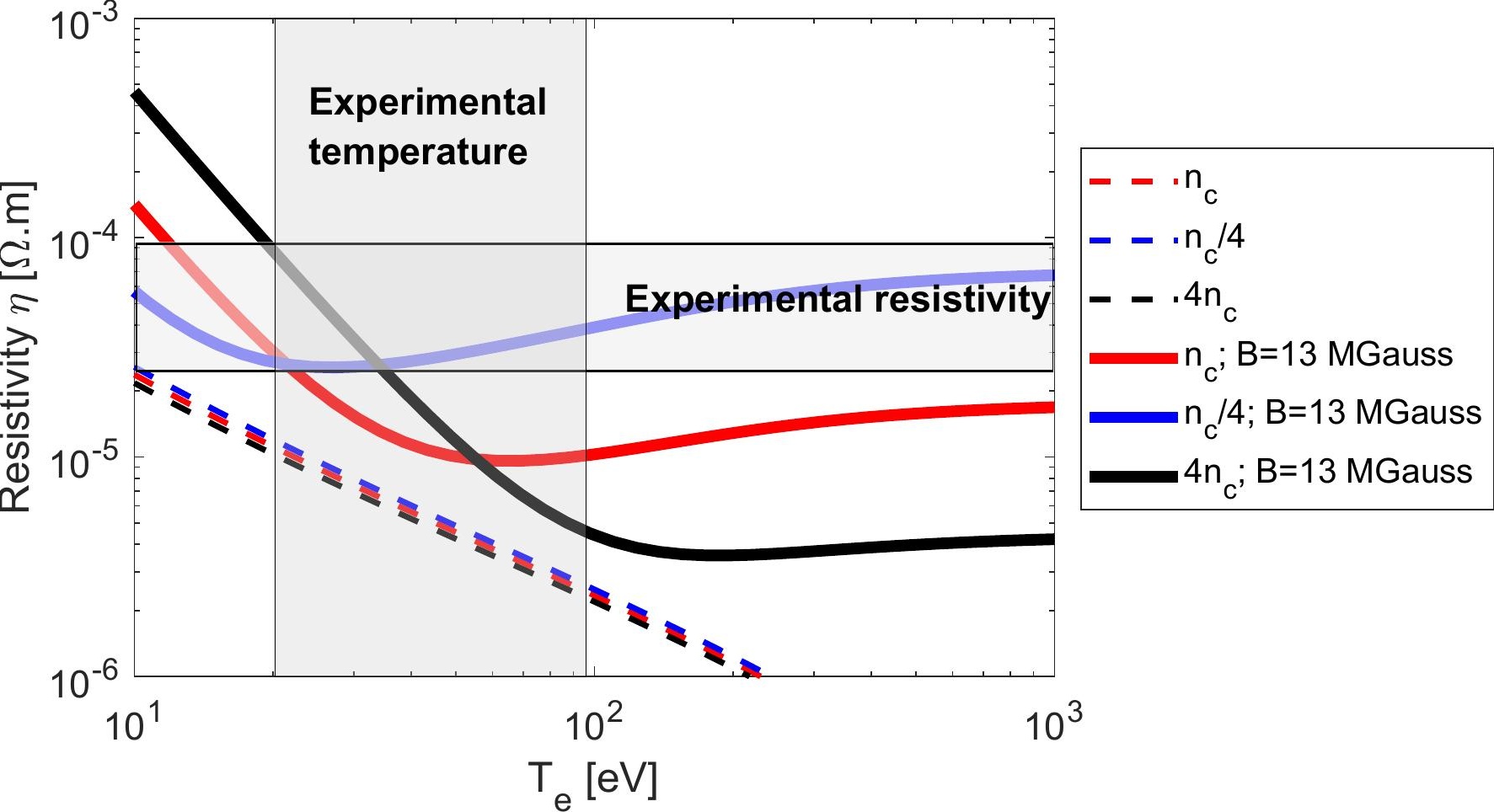

